\title{
Interleukin-17 antagonist attenuates lung inflammation through inhibition of the ERK1/2 and NF-кB pathway in LPS-induced acute lung injury
}

\author{
TIE-JUN LI ${ }^{1}$, LIAN-LI ZHAO ${ }^{2}$, JING QIU $^{2}$, HAI-YAN ZHANG ${ }^{3}$, GUO-XIN BAI $^{2}$ and LIANG CHEN ${ }^{4}$ \\ Departments of ${ }^{1}$ Teaching and ${ }^{2}$ Respiration, Cangzhou Central Hospital; ${ }^{3}$ Department of Nursing, Cangzhou Medical \\ College; ${ }^{4}$ Department of Oncological Surgery, Cangzhou Central Hospital, Cangzhou, Hebei 061001, P.R. China
}

Received March 31, 2016; Accepted April 13, 2017

DOI: $10.3892 / \mathrm{mmr} .2017 .6837$

\begin{abstract}
Interleukin (IL)-17 has been implicated in a variety of inflammatory lung diseases. However, little is known about the expression and biological role of IL-17 in acute lung injury (ALI). Therefore, the aim of the present study was to confirm whether the increase in IL-17 expression following ALI enhances expression of inflammatory cytokines/chemokines through activation of the extracellular signal-regulated kinase $(\mathrm{ERK}) 1 / 2$ and nuclear factor $(\mathrm{NF})-\kappa \mathrm{B}$ signaling pathway in lipopolysaccharide (LPS)-induced acute lung injury; which, in turn, can be blocked by an IL-17 antagonist. The authors indicated that levels of IL-17 mRNA and protein were elevated in the bronchoalveolar lavage fluid (BALF) and lung tissues of ALI rats, and upregulation of IL-17 resulted in the enhanced severity of lung injury. Moreover, treatment with an IL-17 neutralizing antibody significantly inhibited the increases of parameters of ALI in rats, as evidenced by decreased histologic scores, BALF exudate volume, protein leakage and wet-to-dry weight ratio. In addition, management of IL-17 may markedly mitigate LPS-induced pulmonary inflammation, as reflected by the reduced levels of a multitude of proinflammatory cytokines in BALF. Of note, blockade of IL-17 effectively inhibited LPS-induced expression and activation of p-ERK1/2 and nuclear factor (NF)- $\kappa$ B p65 in lung tissues, and suppressed nuclear translocation of NF- $\mathrm{NB}$ p65.
\end{abstract}

Correspondence to: Dr Liang Chen, Department of Oncological Surgery, Cangzhou Central Hospital, 16 Xinhua West Road, Cangzhou, Hebei 061001, P.R. China

E-mail: chenliangsy@163.com

Abbreviations: ALI, acute lung injury; LPS, lipopolysaccharide; IL-17, Interleukin-17; TNF- $\alpha$, tumor necrosis factor- $\alpha$; IL-1 $\beta$, interleukin-1 $\beta$; IL-6, interleukin-6; NF- $\mathrm{B}$, nuclear factor- $\kappa \mathrm{B}$; ERK1/2, extracellular signal-regulated kinase1/2; BALF, bronchoalveolar lavage fluid

Key words: acute lung injury, lung inflammation, lipopolysaccharide, interleukin-17, extracellular signal-regulated kinase $1 / 2$, nuclear factor- $\kappa \mathrm{B}$
These results indicated that IL-17 serves an important role in LPS-induced ALI via stimulation of the ERK1/2 and NF- $\kappa \mathrm{B}$ signaling pathway, and serves as a potential therapeutic target for treating LPS-induced ALI.

\section{Introduction}

Acute lung injury (ALI), a clinical complication associated with respiratory dysfunctions usually a consequence of sepsis and a systemic inflammatory response $(1,2)$. The most severe form of ALI can lead to acute respiratory distress syndrome, respiratory failure, increasing susceptibility to multi-organ dysfunction, and ultimately death with high rates of morbidity and mortality (3). The physiological hallmarks of ALI are a disruption of the alveolar-capillary membrane barrier resulting in non-cardiogenic pulmonary edema, neutrophil and macrophage accumulation, endothelial and epithelial injury, and severe inflammatory response in the lungs and neutrophilic alveolitis $(4,5)$. Despite considerable research, the underlying molecular mechanisms involved in the pathogenesis of acute lung injury and appropriate treatment approaches are still unknown. However, an effective treatment of this life-threatening disease requires a better understanding of the molecular and cellular pathophysiology of ALI.

Lipopolysaccharides (LPS), a pathogenic component of endotoxin released from the cell wall of Gram-negative bacteria, are widely used to induce animal model of ALI $(6,7)$. LPS may induce neutrophil infiltration and the accumulation of pro-inflammatory cytokines, in order to amplify acute lung inflammation (5). A recent study indicated that the binding of LPS to Toll-like receptor 4 caused I $\kappa$ B- $\alpha$ phosphorylation and degradation, activated nuclear factor (NF)- $\kappa$, and subsequently led to the uncontrolled and excessive production of pro-inflammatory mediators, such as tumor necrosis factor (TNF)- $\alpha$, interleukin (IL)-1 $\beta$, IL-6 $(8,9)$. In addition, mitochondrial dysfunction and cell apoptosis participated in the pathological process of LPS-induced ALI, and inhibition of apoptosis decreased lung damage (10). More recently, upregulation expression of IL-17, including IL-17A and IL-17F, were observed in the lung tissues, BALF and serum of ALI rats (11). It is generally believed that IL-17 as a pro-inflammatory cytokine serve a crucial role in triggering inflammatory responses. 
The IL-17 family including six members, designated IL-17A-F, is a pleiotropic pro-inflammatory cytokine $(12,13)$. Growing evidences suggested that IL-17 serve an essential role in a multitude of autoimmune diseases, inflammation and cancer (14-16). Recent evidence indicated that IL-17 may promote epithelial cells to secrete IL-6, GCSF and GM-CSF, which, in turn, recruits neutrophils to the airways (17). Notably, it is reported that TNF- $\alpha$, IL- $1 \beta$, IL- 6 and IL- 8 are the downstream target genes of IL-17 (18). However, the potential role and underlying mechanism of IL-17 in development of LPS-induced ALI and pulmonary inflammation are currently unknown. Therefore, the purpose of the present study was to investigate the expression of IL-17 and possible molecular mechanisms involved in inflammatory response in an ALI rat model by LPS intratracheal instillation.

\section{Materials and methods}

Animals. Male Sprague-Dawley rats, 8-weeks-old and (weight, 200-220 g), were obtained from Vital River Laboratories Co., Ltd. (Beijing, China). All animals used for experiments were allowed free access to food and water, and housed under specific pathogen-free conditions with a $12 \mathrm{~h}$ light/dark cycle in a controlled temperature $\left(20-25^{\circ} \mathrm{C}\right)$ and humidity $(50 \pm 5 \%)$ environment. All experimental protocols were approved by the Laboratory Animal Committee of Hebei Medical University, (Shijiazhuang, China). The rats were sacrificed under anesthesia with chloral hydrate $(350 \mathrm{mg} / \mathrm{kg}$ i.p.), followed by cervical dislocation. All efforts were made to minimize suffering.

Experimental protocol and LPS-induced ALI model. A total of 90 rats were randomly divided into three groups ( $n=12 /$ group): (1) Control group: Received saline by intratracheal instillation $(5 \mathrm{ml} / \mathrm{kg})$ under anesthesia using inhaled isoflurane (Boston Biochem, Inc., Cambridge, MA, USA); (2) ALI group: ALI was induced by intratracheal instillation of LPS to induce acute lung injury model in vivo, as previously described $(5,19)$. In brief, rats were anaesthetized and injected intravenously with LPS ( $5 \mathrm{mg} / \mathrm{kg}$, Sigma-Aldrich; Merck KGaA, Darmstadt, Germany). (3) IL-17 mAb group: Rats were injected intraperitoneally with IL-17 monoclonal antibody (100 $\mu \mathrm{g} / \mathrm{ml}$, IL-17 mAb, Clone \# 50101; R\&D Systems, Inc., Minneapolis, MN, USA). Rats in all groups were sacrificed respectively at 3, 6 and $24 \mathrm{~h}$ following injection, 10 rats per group per time point.

Lung wet/dry (W/D) weight ratio. At $6 \mathrm{~h}$ following LPS or saline challenge, the lung W/D weight ratio was calculated to evaluate the extent of acute pulmonary edema, Five rats per group were anesthetized, harvested and cleaned from blood, weighed to obtain the wet weight, and then placed in an oven for $48 \mathrm{~h}$ at $80^{\circ} \mathrm{C}$ for the measurement of the dry weight. The ratio of the wet weight to dry weight was then calculated (20).

Histopathological evaluation. Morphological changes in the lungs were examined by hematoxylin and eosin (H\&E) staining. At $6 \mathrm{~h}$ and $24 \mathrm{~h}$ following the instillation of LPS, rats were euthanized for histological assessment. The right upper lobes were collected, fixed in $10 \%$ formalin for $48 \mathrm{~h}$ and embedded in paraffin. Sections of fixed embedded tissues
(5 $\mu \mathrm{m}$ thick) were cut on a Leica model 2165 rotary microtome (Leica Microsystems GmbH, Wetzlar, Germany), placed on glass slides, deparaffinized and sequentially stained with H\&E (Thermo Fisher Scientific, Inc., Waltham, MA, USA) for examination under an optical microscope (BX51; Olympus Corporation, Tokyo, Japan). The extents of histological injury were evaluated by two blinded experienced investigators. The degree of lung injury was graded using a scoring system based on histological features, including edema, congestion and hyperemia, tissue infiltration and neutrophil margination, and the presence of intra-alveolar hemorrhage, debris and cellular hyperplasia. Each feature was graded as either absent $=0$, mild $=1$, moderate $=2$, or severe $=3$. The total score was calculated for histopathological evaluation of each rat.

Bronchoalveolar lavage fluid (BALF) collection. BALF was collected by intratracheal intubation as previously described (5). Briefly, after mice were anesthetized, the trachea was exposed and cannulated with a sterile catheter. BAL was performed with a $5 \mathrm{ml} 0.9 \%$ saline solution. The lavage was repeated twice with saline to recover a total volume of 4-5 ml. Lavage samples were centrifuged at 1,000 x g at $4^{\circ} \mathrm{C}$ for $10 \mathrm{~min}$. All samples were stored at $-80^{\circ} \mathrm{C}$ to measure chemokine levels by using ELISA kits.

ELISA. The concentrations of TNF- $\alpha$, IL-6, IL-1 $\beta$ and IL-17 in BALF were detected using cytokine specific Quantikine ELISA kits (TNF- $\alpha$, SEA133Ra; IL-6, SEA079Ra; IL-1 $\beta$, SEA073Ra; IL-17, SEA063Ra; Wuhan USCN Business Co., Ltd., Wuhan, China) according to the manufacturer's instructions. The absorbance was read at $490 \mathrm{~nm}$ on an ELISA plate scanner (Molecular Devices, LLC, Sunnyvale, CA, USA). All experiments are performed at least in triplicate samples and results are presented as the mean value. A standard curve using recombinant cytokine was generated for each assay.

RNA isolation and reverse transcription-quantitative polymerase chain reaction $(R T-q P C R)$ analysis. Total RNA was extracted from rat lung tissues with Tripure Isolation reagent (Thermo Fisher Scientific, Inc.). The qPCR kits (Takara Biotechnology Co., Ltd., Dalian, China) were used for the qPCR experiment. cDNA was synthesized from total RNA using a SuperScript Reverse Transcriptase kit (Thermo Fisher Scientific, Inc.) according to the manufacturer's instructions. The primers of PCR were as follows: Rat IL-17 forward, 5'-ATCCCTCAAAGTTCAGTGTGTCC-3' and reverse, 5'-GGACAATAGAGGAAACGCAGGT-3'; GAPDH forward, 5'-CAAAGTTGTCATGGATGACC-3' and reverse, 5'-CCATGGAGAAGGCTGGGG-3'. RT-qPCR was performed on a Real-Time PCR System (Applied Biosystems; Thermo Fisher Scientific, Inc.; ABI Prism 7300) instrument with SYBR Premix Ex Taq (Takara Bio, Inc., Otsu, Japan), starting with $1 \mathrm{ng}$ reverse-transcribed total RNA. PCR was performed under the following conditions: $95^{\circ} \mathrm{C}$ for $10 \mathrm{~min}$, 40 cycles of $95^{\circ} \mathrm{C}$ for $5 \mathrm{sec}$ and $60^{\circ} \mathrm{C}$ for $30 \mathrm{sec}$. GAPDH was used as an internal control. The relative mRNA expression levels were calculated by the $2^{-\Delta \Delta \mathrm{Cq}}$ method (21).

Western blot analysis. Lung tissues were lysed in total protein cell lysis buffer (Thermo Fisher Scientific, Inc.) supplemented 

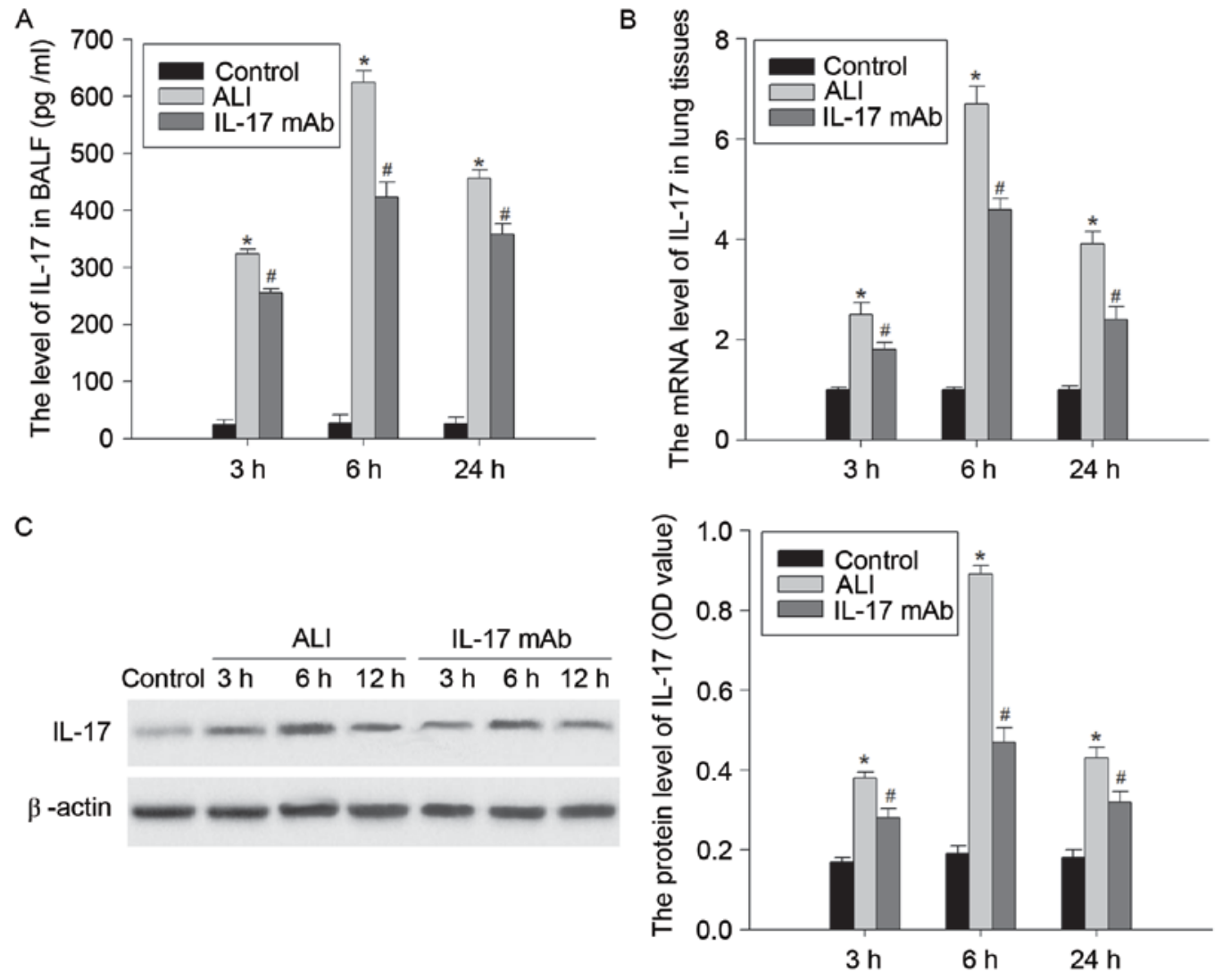

Figure 1. The expression levels of IL-17 in LPS-induced lung injury. Following LPS (5 mg/kg) or saline intravenously injection to rats, BALF and lung tissues were harvested at 3,6 and $24 \mathrm{~h}$. Concentrations of IL-17 in (A) BALF, as well as the (B) mRNA and (C) protein expression of IL-17 were determined in each group. Data are presented as the mean \pm standard deviation of eight independent experiments. ${ }^{*} \mathrm{P}<0.05$ vs. the Control group; ${ }^{*} \mathrm{P}<0.05$ vs. the $\mathrm{ALI}$ group. IL, interleukin; BALF, bronchoalveolar lavage fluid; ALI, acute lung injury; mAb, monoclonal antibody.

with 5\% Proteinase Inhibitor cocktail (Sigma-Aldrich; Merck KGaA), incubated on ice for $30 \mathrm{~min}$, and centrifuged at $15,000 \mathrm{x}$ g for $15 \mathrm{~min}$. Protein concentrations were determined with the bicinchoninic acid protein assay reagents (Nanjing Jiancheng Bioengineering Institute, Nanjing, China). Total protein $(50 \mu \mathrm{g})$ was loaded in each lane, separated using $10 \%$ SDS-PAGE and transferred onto polyvinylidene fluoride membranes using a wet transfer method at room temperature. Nonspecific binding sites were blocked with 5\% BSA for $1 \mathrm{~h}$, then incubated with rabbit anti-rat IL-17 (1:1,000; SC-52567), nuclear factor (NF)- $\mathrm{B}(1: 1,000$; SC-514451), p-extracellular signal-regulated kinase (ERK) 1/2 (1:1,000; SC-136521) and $\beta$-actin polyclonal antibody $(1: 1,000 ; \mathrm{SC}-7210)$ (all from Santa Cruz Biotechnology, Inc., Dallas, TX, USA) overnight at $4^{\circ} \mathrm{C}$. The following day, the membranes were incubated with incubated with horseradish peroxidase conjugated secondary antibody (1:5,000, \#7074S; Cell Signaling Technology, Inc., Danvers, MA, USA). The immunoreactive bands were visualized with an enhanced chemiluminescence (Bio-Rad Laboratories, Inc., Hercules, CA, USA) reagent. Blots were scanned by densitometry, and integrated density of pixels was quantified using Image Quant software (version, 5.2; Molecular Devices, LLC, Sunnyvale, CA, USA).

Statistical analysis. All data in the tests and figures were presented as means \pm standard deviation. The statistical software SPSS software (version, 13.0; SPSS, Inc., Chicago, IL,
USA) was used for data analysis. One-way analysis of variance (ANOVA) followed by Tukey's post hoc test, or a two-tailed unpaired Student's t-test was applied to evaluate statistical significances. Measurements in the intergroup differences at single timepoints were analyzed by an ANOVA, and, if they demonstrated significance, they were further analyzed by the two-tailed unpaired Student's t-test. $\mathrm{P}<0.05$ was considered statistically significant.

\section{Results}

Expression of IL-17 in the lung tissues of ALI rats induced by $L P S$. In order to confirm that the expression of IL-17 is involved in pathophysiologic process of ALI, the authors detected the mRNA and protein levels of IL-17 in the BALF and lung tissues of a LPS-induced ALI rat model at 3, 6 and $24 \mathrm{~h}$ following LPS challenge. As presented in Fig. 1, the protein levels of IL-17 in the BALF and lung tissues were markedly elevated at 3, 6 and $24 \mathrm{~h}$ following LPS injection. Similarly, the mRNA expression of IL-17 in the lung tissues of LPS-treated rats was enhanced. The mRNA and protein levels of L-17 peaked at $6 \mathrm{~h}$, and prolonged the lag time of $24 \mathrm{~h}$. These results indicated that the expression of IL-17 may be closely correlated with development of LPS-induced lung injury.

Blockade of IL-17 alleviated the severity of lung lesion. To determine the roles of IL-17 in LPS-induced lung injury, the 
A
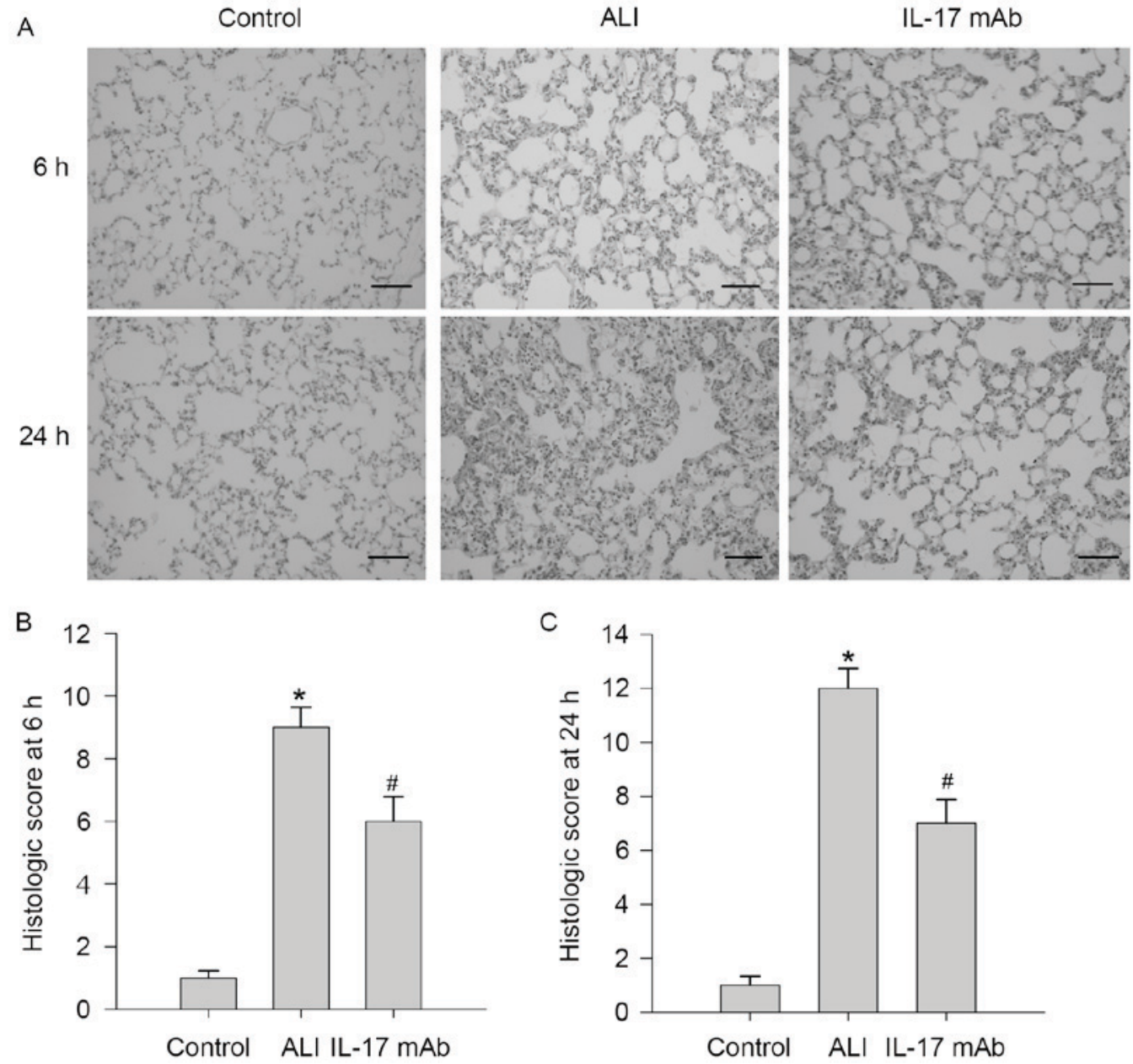

Figure 2. Blockade of IL-17 ameliorates lung histopathological change in lipopolysaccharide-challenged rats. (A) The right upper lobes of lung tissues were prepared, and stained with hematoxylin + eosin for histological analysis evaluation at 6 and $24 \mathrm{~h}$ following ALI. Scale bar: $200 \mu \mathrm{m}$. The slides were assessed using a semi-quantitative scoring system based on the severity of lung injury, respectively for (B) $6 \mathrm{~h}$, and (C) $12 \mathrm{~h}$. Data are presented as the mean \pm standard deviation of eight independent experiments. " $\mathrm{P}<0.05$ vs. the Control group; ${ }^{*} \mathrm{P}<0.05$ vs. the ALI group. IL, interleukin; ALI, acute lung injury; mAb, monoclonal antibody.

authors examined the effects of IL-17 mAb on lung injury. The lung histopathology changes at $6 \mathrm{~h}$ and $24 \mathrm{~h}$ post-ALI are presented in Fig. 2. To observe characteristics of LPS-induced lung injury in rats, LPS treated rats exhibited severe lung injury characterized by inflammation, cell infiltration, interstitial edema, alveolar structural damages and alveolar wall thickening, which was markedly improved by IL-17 mAb treatment, while those phenomena could not be observed in the saline group. A scoring system was used to grade the degree of lung injury, and rats exposed to LPS presented a remarkable increase in lung histologic scores, but the histological scores of IL-17 mAb treated rats were lower than those of ALI group at $6 \mathrm{~h} \mathrm{(Fig.} \mathrm{2B)} \mathrm{and} 24 \mathrm{~h} \mathrm{(Fig.} \mathrm{2C).}$

Blockade of IL-17 attenuated the severity of pulmonary edema. Several well-known hallmarks of ALI-induced pulmonary edema, such as BALF exudate volume (Fig. 3A), protein leakage in BALF (Fig. 3B), and lung W/D weight ratio (Fig. 3C) were remarkably increased in LPS challenge rats compared with those in the control group. Importantly, inhibition of IL-17 using IL-17 mAb, significantly alleviated LPS-induced BALF exudate volume, protein leakage and lung W/D weight ratio. These results demonstrated that
IL-17 mAb treatment may improve pulmonary edema in LPS-challenged rats.

Blockade of IL-17 ameliorated LPS-induced pulmonary inflammation. To confirm the effects of IL-17 mAb treatment on LPS-stimulated expression and release of proinflammatory cytokines and chemokines, the authors measured the levels of IL-17, TNF- $\alpha$, IL-1 $\beta$ and IL- 6 in the BALF of rat by ELISA. As demonstrated in Fig. 4, proinflammatory cytokines including TNF- $\alpha$, IL- $1 \beta$ and IL- 6 were all significantly elevated in BALF in response to LPS treatment. Consistent with this, levels of these proinflammatory factors were dramatically decreased compared with LPS-treated group. These results suggested that IL-17 mAb administration significantly prevented the upregulation of proinflammatory cytokines and chemokines in BALF of LPS challenged rats.

Blockade of IL-17 inhibited LPS-induced ERKI/2 pathway activation. Given the crucial role of IL-17 in the pulmonary inflammation, the authors next sought to investigate the underlying mechanisms of IL-17 involved in the development of ALI. The expression of ERK1/2, p-ERK1/2 in the lung tissues of rats suffering from LPS stimulation was investigated. 

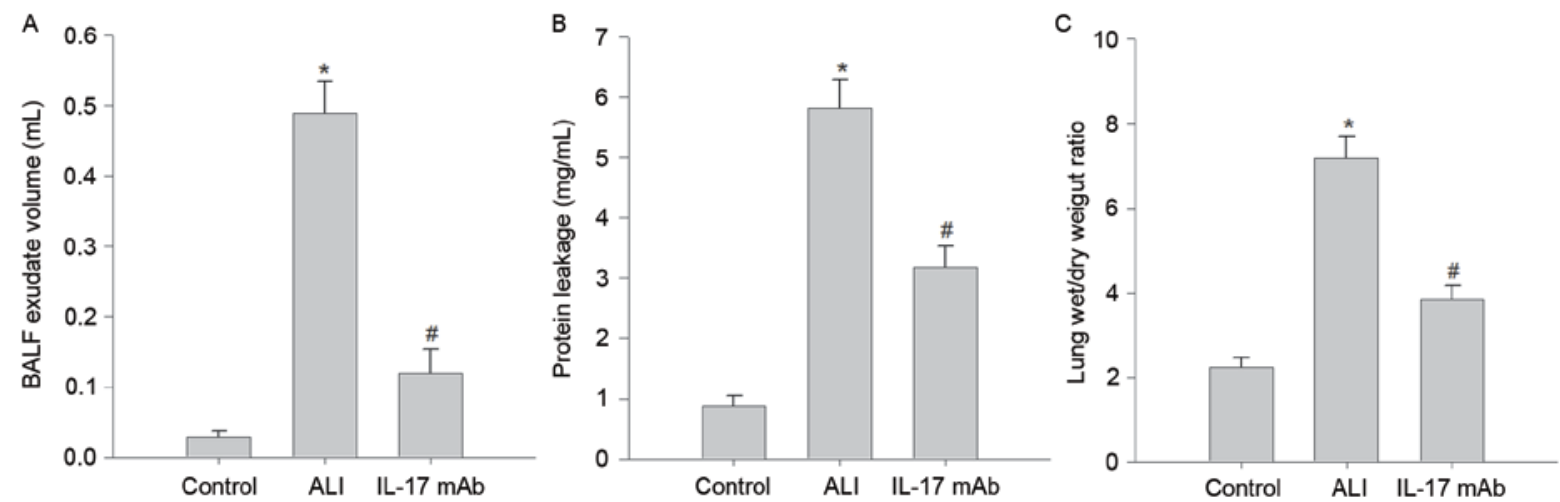

Figure 3. Blockade of IL-17 improves lipopolysaccharide-induced lung edema in rats. BALF were harvested to evaluate the hallmarks of lung edema at $6 \mathrm{~h}$ following ALI. (A) BALF exudate volume. (B) Protein leakage in the BALF. (C) Lung wet/dry weight ratio. Data are presented as the mean \pm standard deviation of eight independent experiments. ${ }^{*} \mathrm{P}<0.05$ vs. the Control group; ${ }^{*} \mathrm{P}<0.05$ vs. the ALI group. IL, interleukin; BALF, bronchoalveolar lavage fluid; ALI, acute lung injury; $\mathrm{mAb}$, monoclonal antibody.
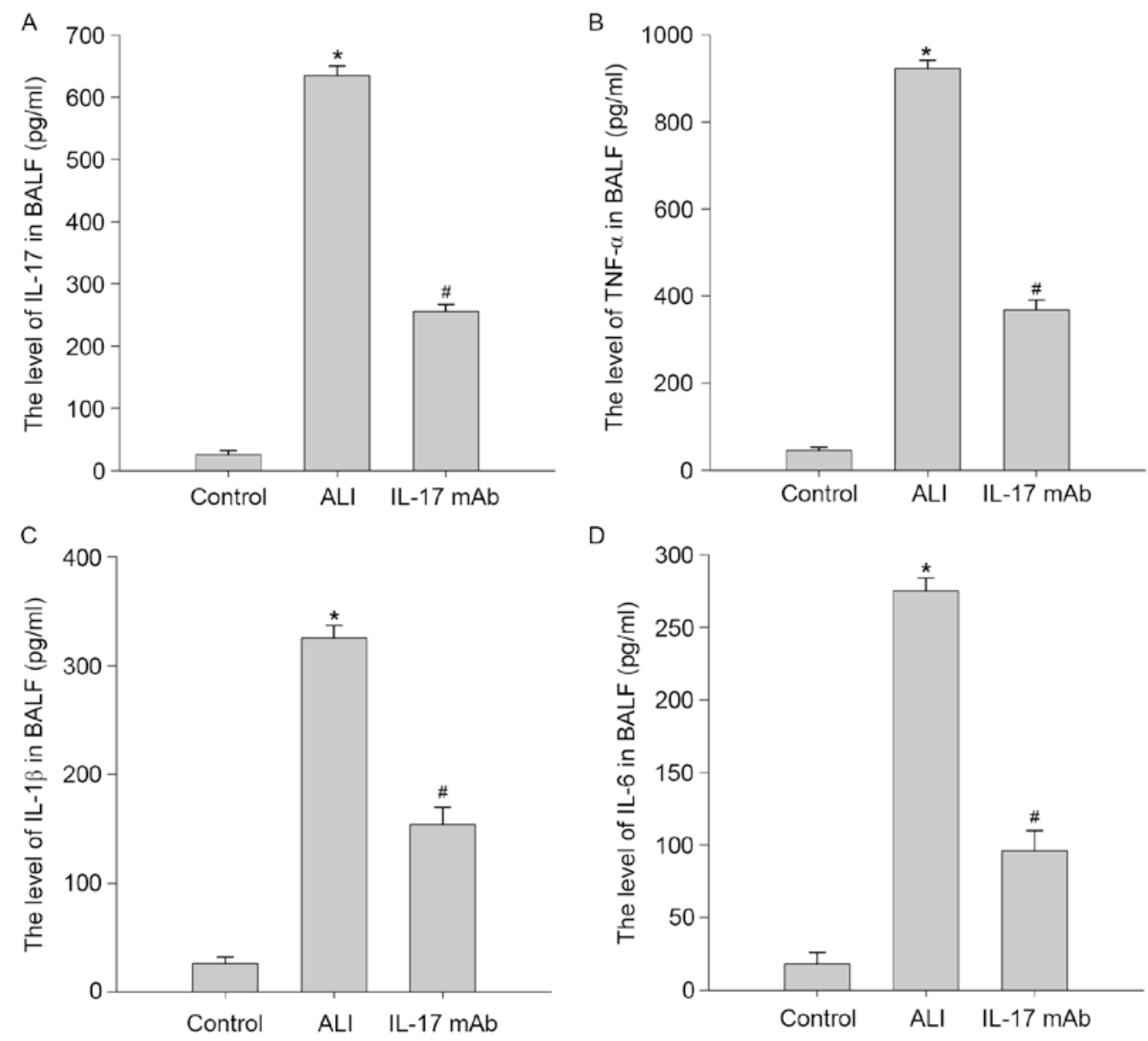

Figure 4. Effects of IL-17 neutralizing antibodies on the level of (A) IL-17, (B) TNF- $\alpha$, (C) IL-1 $\beta$ and (D) IL-6 in bronchoalveolar lavage fluid detected by ELISA at $6 \mathrm{~h}$ following LPS-induced lung injury. Data are presented as the mean \pm standard deviation of eight independent experiments. ${ }^{*} \mathrm{P}<0.05 \mathrm{vs}$. the Control group; ${ }^{\text {P }}<0.05$ vs. the ALI group. IL, interleukin; TNF- $\alpha$, tumor necrosis factor- $\alpha$; mAb, monoclonal antibody; ALI, acute lung injury.

As demonstrated in Fig. 5, the LPS-treated group presented significant increases in the expression of the p-ERK $1 / 2$ protein when compared with the control group. Moreover, the upregulation of p-ERK1/2 protein was reversed by IL-17 $\mathrm{mAb}$ treatment. However, the expression of the ERK1/2 protein presented no significant difference between each group. These findings suggested that effects of IL-17 mAb on the features of
LPS-induced lung inflammation primarily through inhibition of ERK1/2 pathway activity.

Blockade of IL-17 suppressed LPS-induced NF- $\kappa B$ pathway activation. Since NF-KB is known to be a critical transcription factor for inflammation, the molecular mechanisms by which IL-17 mAb administration ameliorates LPS-induced 
A
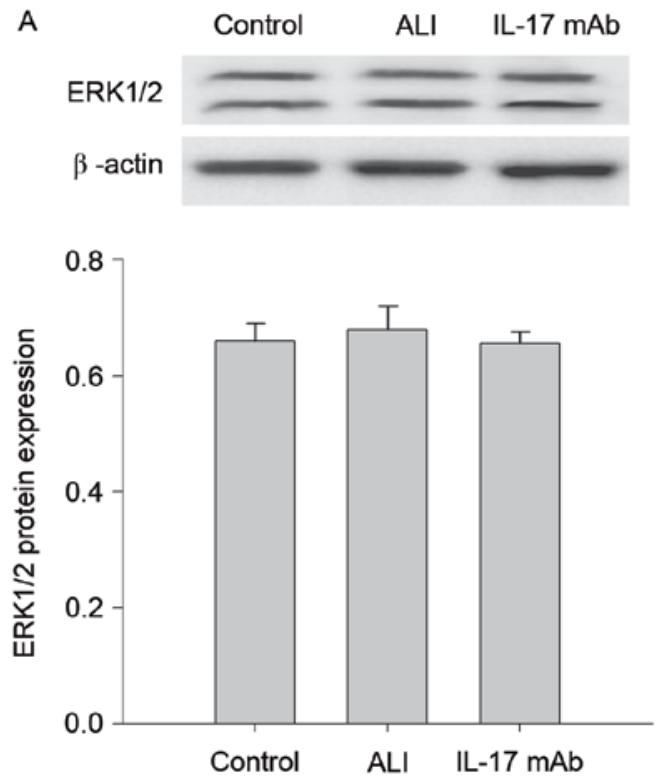

B
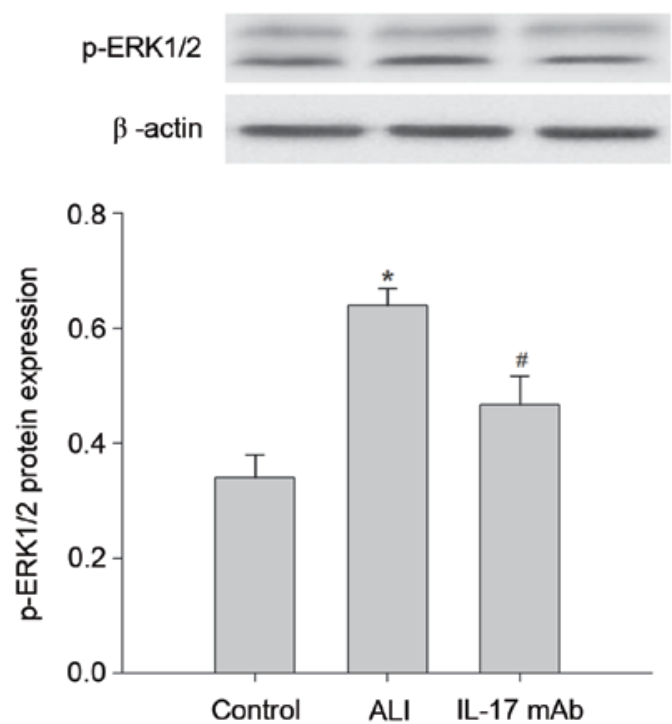

Figure 5. Blockade of IL-17 suppresses the expression and nuclear translocation of NF- $\mathrm{B}$ p65 in the lung of lipopolysaccharide-treated rats. Western blotting was used to analyze the expression of NF- $\mathrm{B}$ p65 in the cytoplasm and nucleus of lung tissues at $6 \mathrm{~h}$ following ALI. (A) Relative protein band densities of $\mathrm{NF}-\kappa \mathrm{B}$ p65 expression in cytoplasm, and the respective densitometric analysis of cytosolic NF- $\kappa \mathrm{B}$ p65 bands, normalized against $\beta$-actin. (B) Relative protein band densities of nuclear NF- $\mathrm{B}$ p 65 expression, and the respective densitometric analysis of nuclear NF- $\kappa \mathrm{B}$ p65 bands, normalized against $\beta$-actin. Data are presented as the mean \pm standard deviation of eight independent experiments. ${ }^{*} \mathrm{P}<0.05$ vs. the Control group; ${ }^{*} \mathrm{P}<0.05$ vs. the $\mathrm{ALI}$ group. IL, interleukin; $\mathrm{NF}$, nuclear factor; ALI, acute lung injury.

A
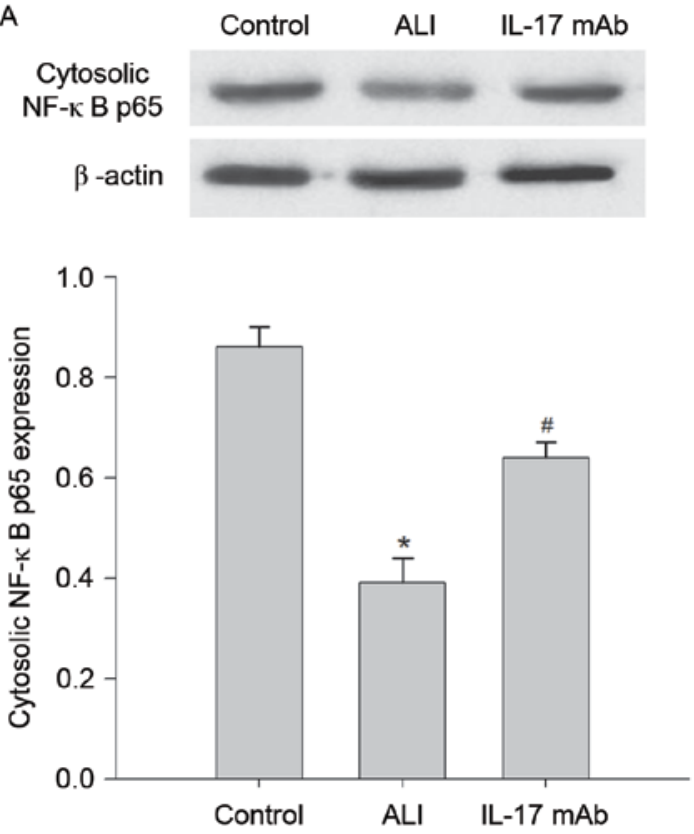

B
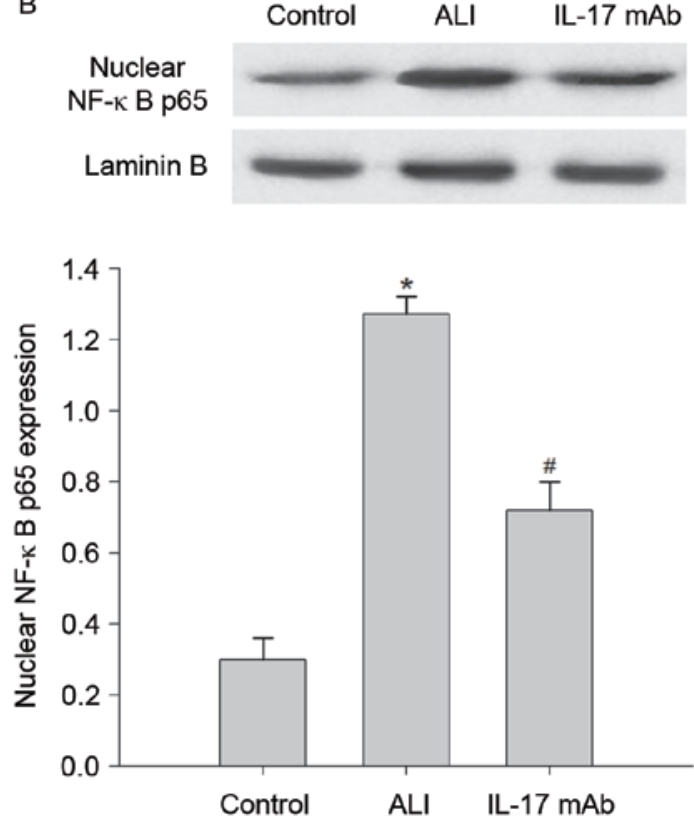

Figure 6. Blockade of IL-17 inhibits the activation of the ERK1/2 pathway in the lung of LPS-induced ALI. Western blotting was used to analyze the expression of ERK1/2 and p-ERK1/2 protein in the lung tissues at $6 \mathrm{~h}$ following ALI. (A) Relative protein band densities of ERK1/2 expression in the lungs, and the respective densitometric analysis of ERK1/2 bands, normalized against $\beta$-actin. (B) Relative protein band densities of nuclear p-ERK1/2 expression, and the respective densitometric analysis of p-ERK1/2 bands, normalized against laminin B. Data are presented as the mean \pm standard deviation of eight independent experiments. ${ }^{*} \mathrm{P}<0.05$ vs. the Control group, ${ }^{\#} \mathrm{P}<0.05$ vs. the ALI group. IL, interleukin; ERK, extracellular signal-regulated kinase; ALI, acute lung injury; $\mathrm{mAb}$, monoclonal antibody; NF, nuclear factor.

lung inflammation we explored. This was conducted by measuring the levels of $\mathrm{NF}-\kappa \mathrm{B}$ p65 in the cytoplasm and nucleus. As presented in Fig. 6, a basal level of NF- $\kappa$ B p65 was observed in the cytoplasm and nuclei of lung samples in the control group. The level of $\mathrm{NF}-\kappa \mathrm{B}$ p 65 in nuclear extracts of lung samples was significantly increased following the instillation of LPS compared with control group, this increase was reduced by administration of IL-17 mAb. In contrast, cytosolic NF- $\kappa$ B p65 expression was decreased in the ALI group in comparison with control group, and IL-17 $\mathrm{mAb}$ treatment could reversed the decrease in NF- $\mathrm{B}$ p 65 protein. Additionally, western blot analyses revealed that 
the LPS-induced nuclear translocation of NF- $\kappa \mathrm{B}$ p65 was restored by administration of IL-17 $\mathrm{mAb}$.

\section{Discussion}

ALI and acute respiratory distress syndrome (ARDS) are the two most severe clinical diseases that account for extensive morbidity and mortality; this is due to lacking effective therapy strategies. However, looking at decades of extensive investigation, the early diagnostic pathogenetic factors, pathogenesis and specific treatment options of ALI remain undefined. Thus, searching and identifying novel therapeutic strategies with protective effect against ALI are an urgent requirement. ALI is primarily characterized by a severe acute inflammatory response in the lungs and neutrophilic alveolitis (4). Particularly, growing evidence suggests that IL-17 may induce the excessive expression and release of pro-inflammatory cytokines from various cells including Th17 cells, macrophages, and natural killer cells (22). In addition, IL-17 has been reported to be necessary for LPS-induced airway neutrophilia (23).

The present study aimed to evaluate the role of IL-17 in LPS-induced ALI, especially focusing on the associated with the ERK1/2 and $\mathrm{NF}-\kappa \mathrm{B}$ signaling pathway. LPS-induced lung injury in rats is frequently used as a model for studying ALI. Firstly, the authors demonstrated that the instillation of LPS induced pathological damage of lung tissues including alveolar distortion, neutrophil recruitment, interstitial edema and disruption of epithelial integrity. Following this, LPS treatment induction of pulmonary edema was observed, as evidenced by the changes in BALF exudate volume, protein leakage and lung W/D weight ratio. In addition, levels of pro-inflammatory cytokines in BALF, such as TNF- $\alpha$, IL-1 $\beta$ and IL-6 were significantly increased in LPS-induced ALI. Furthermore, levels of IL-17 mRNA and protein were elevated in the BALF and lung tissues. Interestingly, treating a rat model of LPS-induced ALI with IL-17 neutralizing antibody markedly reduced these typical manifestations of lung injury including histologic changes, pulmonary edema and lung inflammation. These findings suggested an involvement of IL-17 in the pathophysiological process of LPS-induced lung injury in rats. Herein, the present study provided evidence demonstrating correlations between the increased in IL-17 levels and the severity of lung injury.

More importantly, the authors further explored whether IL-17-induced the expression of cytokines and chemokines are associated with the ERK1/2 and NF- $\mathrm{BB}$ signaling pathway in a rat model of ALI. NF- $\kappa \mathrm{B}$, a critical transcription factor is involved in the regulation of gene expression in numerous pro-inflammatory mediators $(24,25)$. Furthermore, the activated $\mathrm{NF}-\kappa \mathrm{B}$ may, in turn, activate and control inflammatory responses and regulate gene expression of various enzymes involved in inflammation amplification and maintenance (26). These results suggested that the activation of $\mathrm{NF}-\kappa \mathrm{B}$ signal was important in the early inflammatory response. In the present study, the blockade of IL-17 markedly suppressed nuclear translocation of $\mathrm{NF}-\kappa \mathrm{B}$ p 65 , further inhibiting the excessive release of inflammatory factors. Additionally, the elevated p-ERK1/2 in lung tissues of ALI rats was reversed by the IL-17 neutralizing antibody. It is reported that activation of the ERK1/2 signal could stabilize the mRNAs of the IL-17 downstream target genes $(27,28)$. These findings demonstrated that IL-17 drives the ERK1/2 and NF- $\kappa \mathrm{B}$ signaling pathway to trigger and stabilize the transcription of downstream target genes respectively, such as TNF- $\alpha$, IL-1 $\beta$ and IL-6.

In summary, the authors identified the potential role of IL-17 in the development/maintenance of LPS-induced lung inflammation/injury. Elevated IL-17 expression closely correlated to development of ALI and inhibition of IL-17 using neutralizing antibody resulted in attenuating LPS-induced lung inflammation, which was consistent with previous studies. However, the anti-inflammatory mechanisms of the IL-17 neutralizing antibody was elucidated, which was associated with repressing activation of the ERK1/2 and NF- $\kappa \mathrm{B}$ signaling pathway. These findings increased the understanding of how IL-17 affects lung inflammation in ALI, providing promising potential therapeutic targets for clinical ALI.

\section{Acknowledgements}

The authors would like to thank Professor Lian-li Zhao for critical readings of the manuscript. The present study was supported by a grant from the Natural Science Foundation of Hebei, China (grant no. H2015209309) and the Science and Technology Development Project (grant no. 12140209A-31).

\section{References}

1. Sadowitz B, Jain S, Kollisch-Singule M, Satalin J, Andrews P, Habashi N, Gatto LA and Nieman G: Preemptive mechanical ventilation can block progressive acute lung injury. World J Crit Care Med 5: 74-82, 2016.

2. Li C, Bo L, Liu W, Lu X and Jin F: Enteral immunomodulatory diet (Omega-3 Fatty, Acid, $\gamma$-Linolenic Acid and Antioxidant Supplementation) for acute lung injury and acute respiratory distress syndrome: An updated systematic review and metaanalysis. Nutrients 7: 5572-5585, 2015.

3. Imam F, Al-Harbi NO, Al-Harbi MM, Ansari MA, Zoheir KM, Iqbal M, Anwer MK, Al Hoshani AR, Attia SM and Ahmad SF: Diosmin downregulates the expression of $\mathrm{T}$ cell receptors, pro-inflammatory cytokines and NF- $\kappa \mathrm{B}$ activation against LPS-induced acute lung injury in mice. Pharmacol Res 102: 1-11, 2015.

4. Fu J, Wang Y, Zhang J, Wu W, Chen X and Yang Y: Anti-inflammatory and anti-apoptotic effects of oxysophoridine on lipopolysaccharide-induced acute lung injury in mice. Am J Transl Res 7: 2672-2682, 2015.

5. Wang YY, Qiu XG and Ren HL: Inhibition of acute lung injury by rubriflordilactone in LPS-induced rat model through suppression of inflammatory factor expression. Int J Clin Exp Pathol 8: 15954-15959, 2015.

6. Yan Z, Xiaoyu Z, Zhixin S, Di Q, Xinyu D, Jing X, Jing H, Wang D, Xi Z, Chunrong Z and Daoxin W: Rapamycin attenuates acute lung injury induced by LPS through inhibition of Th17 cell proliferation in mice. Sci Rep 6: 20156, 2016.

7. Gross CM, Rafikov R, Kumar S, Aggarwal S, Ham PB III, Meadows ML, Cherian-Shaw M, Kangath A, Sridhar S, Lucas R and Black SM: Endothelial nitric oxide synthase deficient mice are protected from lipopolysaccharide induced acute lung injury. PLoS One 10: e0119918, 2015.

8. Xu C, Chen G, Yang W, Xu Y, Xu Y, Huang X, Liu J, Feng Y, $\mathrm{Xu}$ Y and Liu B: Hyaluronan ameliorates LPS-induced acute lung injury in mice via Toll-like receptor (TLR) 4-dependent signaling pathways. Int Immunopharmacol 28: 1050-1058, 2015.

9. Feng G, Jiang ZY, Sun B, Fu J and Li TZ: Fisetin alleviates lipopolysaccharide-induced acute lung injury via TLR4-Mediated NF- $\mathrm{B}$ signaling pathway in rats. Inflammation 39: 148-157, 2016.

10. Aggarwal S, Dimitropoulou C, Lu Q, Black SM and Sharma S: Glutathione supplementation attenuates lipopolysaccharide-induced mitochondrial dysfunction and apoptosis in a mouse model of acute lung injury. Front Physiol 3: 161, 2012. 
11. You QH, Zhang D, Niu CC, Zhu ZM, Wang N, Yue Y and Sun GY: Expression of IL-17A and IL-17F in lipopolysaccharide-induced acute lung injury and the counteraction of anisodamine or methylprednisolone. Cytokine 66: 78-86, 2014.

12. Gong F, Liu Z, Liu J, Zhou P, Liu Y and Lu X: The paradoxical role of IL-17 in atherosclerosis. Cell Immunol 297: 33-39, 2015.

13. Khan D and Ansar Ahmed S: Regulation of IL-17 in autoimmune diseases by transcriptional factors and microRNAs. Front Genet 6: 236, 2015.

14. Kugyelka R, Kohl Z, Olasz K, Mikecz K, Rauch TA, Glant TT and Boldizsar F: Enigma of IL-17 and Th17 cells in rheumatoid arthritis and in autoimmune animal models of arthritis. Mediators Inflamm 2016: 6145810, 2016.

15. Qian X, Chen H, Wu X, Hu L, Huang Q and Jin Y: Interleukin-17 acts as double-edged sword in anti-tumor immunity and tumorigenesis. Cytokine 89: 34-44, 2017.

16. Beringer A, Noack M and Miossec P: IL-17 in chronic inflammation: From discovery to targeting. Trends Mol Med 22: 230-241, 2016.

17. Li Q, Gu Y, Tu Q, Wang K, Gu X and Ren T: Blockade of Interleukin-17 restrains the development of acute lung injury. Scand J Immunol 83: 203-211, 2016.

18. Onishi RM and Gaffen SL: Interleukin-17 and its target genes: Mechanisms of interleukin-17 function in disease. Immunology 129: 311-321, 2010.

19. Matute-Bello G, Frevert CW and Martin TR: Animal models of acute lung injury. Am J Physiol Lung Cell Mol Physiol 295: L379-L399, 2008

20. Xie K, Yu Y, Pei Y, Hou L, Chen S, Xiong L and Wang G: Protective effects of hydrogen gas on murine polymicrobial sepsis via reducing oxidative stress and HMGB1 release. Shock 34: 90-97, 2010.
21. Livak KJ and Schmittgen TD: Analysis of relative gene expression data using real-time quantitative PCR and the 2(-Delta Delta C(T)) Method. Methods 25: 402-408, 2001

22. Shabgah AG, Fattahi E and Shahneh FZ: Interleukin-17 in human inflammatory diseases. Postepy Dermatol Alergol 31: 256-261, 2014.

23. Ferretti S, Bonneau O, Dubois GR, Jones CE and Trifilieff A: IL-17, produced by lymphocytes and neutrophils, is necessary for lipopolysaccharide-induced airway neutrophilia: IL-15 as a possible trigger. J Immunol 170: 2106-2112, 2003.

24. Shih RH, Wang CY and Yang CM: NF-kappaB signaling pathways in neurological inflammation: A mini review. Front Mol Neurosci 8: 77, 2015

25. Zhang $\mathrm{H}$ and Sun SC: NF- $\mathrm{BB}$ in inflammation and renal diseases. Cell Biosci 5: 63, 2015.

26. Schuliga M: NF-kappaB signaling in chronic inflammatory airway disease. Biomolecules 5: 1266-1283, 2015.

27. Hata K, Andoh A, Shimada M, Fujino S, Bamba S, Araki Y, Okuno T, Fujiyama Y and Bamba T: IL-17 stimulates inflammatory responses via NF-kappaB and MAP kinase pathways in human colonic myofibroblasts. Am J Physiol Gastrointest Liver Physiol 282: G1035-G1044, 2002.

28. Bulek K, Liu C, Swaidani S, Wang L, Page RC, Gulen MF, Herjan T, Abbadi A, Qian W, Sun D, et al: The inducible kinase IKKi is required for IL-17-dependent signaling associated with neutrophilia and pulmonary inflammation. Nat Immunol 12: 844-852, 2011 